\title{
BOUNDED NEGATIVITY AND HARBOURNE CONSTANTS ON RULED SURFACES
}

\author{
KRISHNA HANUMANTHU AND ADITYA SUBRAMANIAM
}

\begin{abstract}
Let $X$ be a smooth projective surface and let $\mathcal{C}$ be an arrangement of curves on $X$. The Harbourne constant of $\mathcal{C}$ was defined as a way to investigate the occurrence of curves of negative self-intersection on blow ups of $X$. This is related to the bounded negativity conjecture which predicts that the self-intersection number of all reduced curves on a surface is bounded below by a constant. We consider a geometrically ruled surface $X$ over a smooth curve and give lower bounds for the Harbourne constants of transversal arrangements of curves on $X$. We also define a global Harbourne constant as the infimum of Harbourne constants for arrangements of a specific type and give a lower bound for it.
\end{abstract}

\section{InTRODUCTION}

Let $X$ be a smooth complex projective surface. $X$ is said to have bounded negativity if there exists an integer $b(X)$, depending only on $X$, such that $C^{2} \geq-b(X)$ for all reduced curves $C$ on $X$. The Bounded Negativity Conjecture $(B N C)$ asserts that every smooth complex projective surface has bounded negativity. To verify BNC, it suffices to show that selfintersection of reduced and irreducible curves is bounded below, by [4, Proposition 5.1]. While it is easy to prove this conjecture in some cases (for example, when the anti-canonical divisor $-K_{X}$ is effective, it follows from adjunction formula), it is open in general. For example, the conjecture is open for surfaces obtained by blowing up at least ten points on the complex projective plane $\mathbb{P}_{\mathbb{C}}^{2}$.

The notion of Harbourne constants was defined in 3 in an attempt to understand and clarify the bounded negativity conjecture. To illustrate the concept, consider the blow up $X$ of $\mathbb{P}_{\mathbb{C}}^{2}$ at $r$ distinct points. It is clear that the occurrence of negative curves on $X$ depends on the position of the points that are blown up. For example, if the points are general enough, it is conjectured that $C^{2} \geq-1$ for all reduced and irreducible curves $C \subset X$. On the other hand, $C^{2}=1-r$ if the points are collinear and $C$ is the strict transform of the line containing them. The key idea is to divide by $r$ and consider the ratio $C^{2} / r$ for all reduced, not necessarily irreducible, curves $C$ on $X$. The problem then is to bound these ratios $C^{2} / r$. The infimum of these ratios as we vary the points on $\mathbb{P}^{2}$ and the reduced curves on blow ups of $\mathbb{P}^{2}$ is an invariant called the global Harbourne constant of $\mathbb{P}^{2}$ and it is denoted by $H\left(\mathbb{P}^{2}\right)$. It is not known if $H\left(\mathbb{P}^{2}\right) \neq-\infty$. But if $H\left(\mathbb{P}^{2}\right) \neq-\infty$, then BNC holds for a blow up of $\mathbb{P}^{2}$ at any finite set of points. One can similarly define the invariant $H(X)$ for any surface $X$ and

Date: February 17, 2020.

2010 Mathematics Subject Classification. 14C20, 14C17.

The first author was partially supported by DST SERB MATRICS grant MTR/2017/000243. Both authors were partially supported by a grant from Infosys Foundation. 
if $H(X) \neq-\infty$, then BNC holds for blow ups of $X$ at finite sets of points; see [3, Remark $2.3]$.

In order to understand the global Harbourne constant $H(X)$ of a surface $X$, it is natural to consider the following situation. Let $\mathcal{C}=\left\{C_{1}, \ldots, C_{d}\right\}$ be an arrangement of irreducible and reduced curves on $X$. Let $D$ be the effective divisor $C_{1}+\ldots+C_{d}$ on $X$. Let $\tilde{X} \rightarrow X$ be the blow up of $X$ at the singular points $p_{1}, \ldots, p_{r}$ of $D$ and let $\tilde{D}$ be the strict transform of $D$. We are interested in the ratio $\frac{\tilde{D}^{2}}{r}$. As we vary the arrangements $\mathcal{C}$ on $X$ and take the infimum of $\frac{\tilde{D}^{2}}{r}$, we obtain $H(X)$. So it is natural to first try to bound $H(\mathcal{C})=H(D):=\frac{\tilde{D}^{2}}{r}$, for a specific reduced curve $D$.

This problem is studied in [3] when $X=\mathbb{P}^{2}$ and all the irreducible components of $D$ are lines. We say in this case that $\mathcal{C}$ is a line arrangement. [3, Theorem 3.3] proves that $H(D)>-4$ for all such $D$.

Harbourne constants for arrangements of $d$ lines in $\mathbb{P}_{k}^{2}$ for arbitrary fields $k$ are studied in [5]. The absolute linear Harbourne constant $H(d)$ is defined as the minimum of Harbourne constants of $d$ lines in $\mathbb{P}_{k}^{2}$ as $k$ varies over all fields. The value of $H(d)$ is computed for small values of $d$ and also special forms of $d$. See [5, Theorem 1.4, Theorem 1.6].

The case of arrangements of conics on $\mathbb{P}^{2}$ was studied in [22]. It is proved in [22, Theorem A] that $H(\mathcal{C}) \geq-4.5$ for any such arrangement $\mathcal{C}$.

The author of [23] considers arrangements $\mathcal{C}$ of elliptic curves on an abelian surface or on $\mathbb{P}^{2}$. It is proved that $H(\mathcal{C}) \geq-4$. Further, in [23, Theorem 5], a sequence of reduced curves $D_{n} \subset \mathbb{P}^{2}$ (each of which is a union of elliptic curves) is constructed such that $\lim _{n} H\left(D_{n}\right)=$ -4 .

In [21], the authors consider reduced divisors $D=C_{1}+\ldots+C_{d}$ on $\mathbb{P}^{2}$, where $C_{i}$ are smooth irreducible plane curves of degree $n \geq 3$ such that $C_{i}$ and $C_{j}$ meet transversally for all $i \neq j$. Assume also that $d \geq 4$ and that there are no points in which all the curves meet. Let $s$ be the number of singular points of $D$. Then they show in [21, Theorem 4.2] that $H(\mathcal{C}) \geq-4+\frac{9 n d-5 n^{2} d}{2 s}$.

Let $X$ be a smooth hypersurface of degree $d \geq 3$ in $\mathbb{P}^{3}$. The Harbourne constants for line arrangements on $X$ were first studied in [18]. The bounds obtained there were generalized in [15]. By [15, Theorem 3.2], the Harbourne constants of line arrangements $\mathcal{C}$ on $X$ satisfy $H(\mathcal{C}) \geq-d(d-1)$ when $d \geq 4$.

Harbourne constants for transversal arrangements of smooth curves on a surface $X$ with numerically trivial canonical class were studied in [14]. The bounds on Harbourne constants were given in terms of the number of curves and the second Chern class of $X$. This bound was generalized to surfaces with non-negative Kodaira dimension in [15].

As the above survey of the literature illustrates, most of the work on Harbourne constants for curve arrangements considered surfaces of non-negative Kodaira dimension or $\mathbb{P}^{2}$. In this paper we look at curve arrangements on ruled surfaces and prove lower bounds on their Harbourne constants. 
The basic tool in studying Harbourne constants for curve arrangements on surfaces is a method developed by Hirzebruch in [10. The idea is to consider a branched abelian covering $Z$ of $X$ branched along the given configuration $\mathcal{C}$. Then consider the desingularization $Y$ of $Z$. Under some conditions on the surface $X$ and the arrangement $\mathcal{C}, Y$ turns out to have nonnegative Kodaira dimension. Then one considers Hirzebruch-Miyaoka-Sakai type inequalities involving the Chern numbers of $Y$. Hirzebruch described the Chern numbers of $Y$ in terms of certain invariants of the surface $X$ and certain combinatorial invariants of the arrangement $\mathcal{C}$. In the end, one obtains inequalities on combinatorial invariants of $\mathcal{C}$ which can then be used to obtain bounds on Harbourne constants.

Hirzebruch [10] carried out this procedure for $X=\mathbb{P}_{\mathbb{C}}^{2}$ and for a line arrangement $\mathcal{C}$ on $X$ to compute the Chern numbers of $Y$. In this case, he showed that

$$
t_{2}+\frac{3}{4} t_{3} \geq d+\sum_{k \geq 5}(k-4) t_{k}, \text { if } t_{d}=t_{d-1}=0
$$

where $d$ is the number of lines in $\mathcal{C}$ and $t_{i}$ is the number of points where exactly $i$ of the lines in $\mathcal{C}$ meet. Using this inequality crucially, the authors of [3] obtain their lower bound on the Harbourne constant of line arrangements in $\mathbb{P}^{2}$ which is mentioned above. In all the known results on Harbourne constants, a Hirzebruch-type inequality is used to obtain a bound for the Harbourne constants.

An interesting question in this situation is to determine whether the surface $Y$ constructed by the method described above is a ball quotient. These are minimal surfaces of general type whose universal cover is the 2-dimensional unit ball. Equivalently, they are minimal surfaces of general type for which the Bogomolov-Miyaoka-Yau inequality is an equality. In other words, a minimal surface $Y$ is a ball quotient if and only if $K_{Y}$ is nef and big and $K_{Y}^{2}=3 e(Y)$, where $K_{Y}$ is the canonical divisor of $Y$ and $e(Y)$ is the topological Euler characteristic of $Y$. In [10], Hirzebruch was interested in constructing ball quotients by starting with line arrangements on $\mathbb{P}^{2}$. We show that the surfaces we construct starting with curve arrangements on ruled surfaces do not give new examples of ball quotients. We follow the methods developed in [2].

The paper is organized as follows.

In Section 2, we recall some basic facts of ruled surfaces and introduce the curve arrangements that we study. We also include some well-known combinatorial properties of these curve arrangements that we require.

In Section [3, using a result of Namba, we construct an abelian cover $Z \rightarrow X$ branched on the given curve arrangement and then consider the desingularization $Y \rightarrow Z$; see Figure 1, We also compute the Chern numbers of $Y$ and relate these to the combinatorial data of the curve arrangement on $X$.

In Section 4, we first show that $Y$ has non-negative Kodaira dimension which enables us to apply a Hirzebruch-Miyaoka-Sakai type inequality. Using this, we prove our main results Theorem 4.7 and Corollary 4.11 about Harbourne constants for curve arrangements on ruled surfaces. Theorem 4.7 gives a lower bound for Harbourne constants for a specific curve 
arrangement on a ruled surface $X$. For a fixed pair of integers $a, b$, we define a global Harbourne constant $H_{a, b}(X)$ which is obtained by taking the infimum of Harbourne constants as the curve arrangements vary (see Definition 4.9). In Corollary 4.11, we give lower bound for global Harbourne constants on any ruled surface. Assuming that the curves in our arrangement do not intersect the normalized section of the ruled surface, we obtain a better bound for the Harbourne constant in Proposition 4.8. Using these bounds, we give a lower bound in Corollary 4.12 for the self-intersection of the strict transform of the curve arrangement for the blow up of all its singular points.

Finally, in Section 5, we show that the surface $Y$ is not a ball quotient (see Theorem 5.2).

We work throughout over the complex number field $\mathbb{C}$.

\section{Preliminaries}

Definition 2.1 (Transversal arrangement). Let $\mathcal{C}=\left\{C_{1}, C_{2}, \ldots, C_{d}\right\}$ be an arrangement of curves on a smooth projective surface $X$. We say that $\mathcal{C}$ is a transversal arrangement if $d \geq 2$, all curves $C_{i}$ are smooth and they intersect pairwise transversally.

Given a transversal arrangement $\mathcal{C}=\left\{C_{1}, C_{2}, \ldots, C_{d}\right\}$, we have a divisor $D=\sum_{i=1}^{d} C_{i}$ on $X$. We use the arrangement $\mathcal{C}$ and the divisor $D$ interchangeably.

Let $\operatorname{Sing}(\mathcal{C})$ be the set of all intersection points of the curves in a transversal arrangement $\mathcal{C}$. Note that $\operatorname{Sing}(\mathcal{C})$ is precisely the set of singularities of the reduced curve $D$, since all the irreducible components of $D$ are nonsingular by hypothesis. Let $s$ denote the number of points in the set $\operatorname{Sing}(\mathcal{C})$.

Definition 2.2 (Combinatorial invariants of transversal arrangements). Let $\mathcal{C}$ be a transversal arrangement on a smooth surface $X$. For a point $p \in X$, let $r_{p}$ denote the number of elements of $\mathcal{C}$ that pass through $p$. We call $r_{p}$ the multiplicity of $p$ in $\mathcal{C}$. We say $p$ is a $k$-fold point of $\mathcal{C}$ if there are exactly $k$ curves in $\mathcal{C}$ passing through $p$. For a positive integer $k \geq 2$, $t_{k}$ denotes the number of $k$-fold points in $\mathcal{C}$.

These numbers satisfy the following standard equality, which follows by counting incidences in a transversal arrangement in two ways:

$$
\sum_{i<j}\left(C_{i} \cdot C_{j}\right)=\sum_{k \geq 2}\left(\begin{array}{l}
k \\
2
\end{array}\right) t_{k} .
$$

Also, let

$$
f_{i}=f_{i}(D):=\sum_{k \geq 2} k^{i} t_{k}
$$

In particular, $f_{0}=s$ is the number of points in $\operatorname{Sing}(\mathcal{C})$.

Definition 2.3 (Harbourne constants of a transversal arrangement). Let $X$ be a smooth projective surface. Let $D=\sum_{i=1}^{d} C_{i}$ be a transversal arrangement of curves on $X$ with $s=$ 
$s(D)>0$. The rational number

$$
H(X, \mathcal{C})=H(X, D)=\frac{1}{s}\left(D^{2}-\sum_{P \in \operatorname{Sing}(D)} r_{P}^{2}\right)
$$

is called the Harbourne constant of the transversal arrangement $\mathcal{C}$.

When the surface $X$ is clear from the context, we simply write $H(\mathcal{C})$ or $H(D)$ to denote the Harbourne constants.

In this paper, we consider transversal arrangements of curves on ruled surfaces. We follow the notation in [7, Chapter V, Section 2].

Let $C$ be a smooth complex curve of genus $g$. A geometrically ruled surface is a surface of the form $X=\mathbb{P}_{C}(E)$ where $E$ is a rank 2 vector bundle on $C$. We refer to such surfaces simply as ruled surfaces. Let $\phi: X \rightarrow C$ be the natural map.

Note that $\mathbb{P}_{C}(E) \cong \mathbb{P}_{C}(E \otimes \mathcal{L})$ for any line bundle $\mathcal{L}$ on $C$. Let $E$ be a normalized vector bundle with $X=\mathbb{P}_{C}(E)$; this means that $H^{0}(C, E) \neq 0$ and $H^{0}(C, E \otimes \mathcal{L})=0$ for all line bundles $\mathcal{L}$ on $C$ with $\operatorname{deg}(\mathcal{L})<0$. We set $e:=\operatorname{deg}\left(\wedge^{2} E\right)$. This invariant is uniquely determined by $X$.

We fix a section $C_{0}$ of $X$ with $\mathcal{L}\left(C_{0}\right)=\mathcal{O}_{\mathbb{P}(E)}(1)$. Let $f$ denote the numerical class of a fiber of $\phi$. Then any element of $\operatorname{Num}(X)$ has the form $a C_{0}+b f$ for $a, b \in \mathbb{Z}$. The intersection product on $\operatorname{Num}(X)$ is determined by $C_{0}^{2}=-e, C_{0} \cdot f=1$ and $f^{2}=0$. Any canonical divisor on $X$, denoted by $K_{X}$, is numerically equivalent to $-2 C_{0}+(2 g-2-e) f$.

Let $X$ be a ruled surface over a smooth complex curve $C$ of genus $g$ with $e \geq 0$. If an irreducible curve on $X$, different from $C_{0}$ and $f$, is numerically equivalent to $a C_{0}+b f$, then $a>0$ and $b \geq a e$. A divisor on $X$ which is numerically equivalent to $a C_{0}+b f$ is ample if and only if $a>0$ and $b>a e$.

For more details, see [7, Chapter V, Section 2].

Assumption 2.4. Let $X$ be a ruled surface over a smooth curve of genus $g \geq 0$ with invariant $e=e(X) \geq 4$. Let $\mathcal{C}=\left\{C_{1}, C_{2}, \ldots, C_{d}\right\}$ be a transversal arrangement of curves on $X$ with $d \geq 4$ and $t_{d}=0$. Suppose that all the curves $C_{i}$ in $\mathcal{C}$ are linearly equivalent to a fixed divisor $A$ on $X$, where $A$ is numerically equivalent to $a C_{0}+b f$, for $a, b \in \mathbb{Z}$ with $a>0$ and $b \geq a e$. Note that under these assumptions, $C_{i} \cdot C_{j}=2 a b-a^{2} e$ for all curves $C_{i}, C_{j} \in \mathcal{C}$.

Lemma 2.5. Let $\mathcal{C}=\left\{C_{1}, C_{2}, \ldots, C_{d}\right\}$ be a transversal arrangement of curves on a ruled surface $X$ satisfying Assumption 2.4. Then we have the following.

(1) For every curve $C_{i} \in \mathcal{C}$, we have $\sum_{p \in C_{i}}\left(r_{p}-1\right)=\left(2 a b-a^{2} e\right)(d-1)$.

(2) $f_{2}-f_{1}=\sum_{k \geq 2} k(k-1) t_{k}=\left(2 a b-a^{2} e\right) d(d-1)$.

Proof. First we prove (1). Given a multiple point $p \in C_{i}, r_{p}-1$ is the number of curves of the arrangement passing through $p$ different from $C_{i}$. As every curve meets every other curve in 
$2 a b-a^{2} e$ distinct points, the expression $\sum_{p \in C_{i}}\left(r_{p}-1\right)$ counts all curves of the arrangement different from $C_{i}, 2 a b-a^{2} e$ times each. So (1) holds.

The first equality in (2) follows from the definition of $f_{2}, f_{1}$. As $\sum_{C_{i} \in \mathcal{C}} \sum_{p \in C_{i}}\left(r_{p}-1\right)=$ $\sum_{k \geq 2} k(k-1) t_{k}$, the second equality in (2) follows from (1).

\section{Construction of the abelian cover}

Our arguments follow the model developed by Hirzebruch in 10. These ideas have been used by several recent authors. See [6, 18, 21, 22, 23], for example.

Let $X$ be a ruled surface over a smooth curve $C$ of genus $g$. Let $\mathcal{C}=\left\{C_{1}, \ldots, C_{d}\right\}$ be a transversal arrangement of curves on $X$ satisfying Assumption 2.4. Our goal is to give bounds for the Harbourne constant $H(X, \mathcal{C})$. The starting point is to consider a branched covering of $X$ branched along the curves in $\mathcal{C}$. In order to prove that such a branched covering does in fact exist for the ruled surface $X$, we use a result of Namba, which we recall below.

As above, let $D=\sum_{i=1}^{d} C_{i}$. Let $\operatorname{Div}(X, D)$ be the subgroup of the $\mathbb{Q}$-divisors on $X$ generated by all the integral divisors and the following $\mathbb{Q}$-divisors: $\frac{C_{1}}{2}, \frac{C_{2}}{2}, \ldots, \frac{C_{d}}{2}$.

Let $\sim$ be linear equivalence in $\operatorname{Div}(X, D)$, where $G \sim G^{\prime}$ if and only if $G-G^{\prime}$ is an integral principal divisor. Let $\operatorname{Div}^{0}(X, D) / \sim$ denote the kernel of the first Chern class map:

$$
\begin{array}{ccc}
\operatorname{Div}(X, D) / \sim & \rightarrow & H^{1,1}(X, \mathbb{R}) \\
G & \mapsto & c_{1}(G)
\end{array}
$$

We use the following result of Namba [16, Theorem 2.3.20]. In our special case, it says the following.

Theorem 3.1 (Namba). There exists a finite abelian cover $Z \rightarrow X$ with branch locus equal to $D$ and ramification index 2 at each $C_{i}$ if and only if for every $j=1, \ldots, d$, there exists an element of finite order $v_{j}=\sum \frac{a_{i j}}{2} C_{i}+E_{j}$ of $D_{i v}^{0}(X, D) / \sim$, where $E_{j}$ are integral divisors and $a_{j j} \in \mathbb{Z}$ is odd for every $j=1, \ldots, d$.

In this case, the subgroup of $\operatorname{Div}^{0}(X, D) / \sim$ generated by the $v_{j}$ is isomorphic to the Galois group of the abelian cover $Z \rightarrow X$.

Set $v_{1}=v_{2}=\frac{C_{1}-C_{2}}{2}$ and $v_{j}=\frac{C_{1}-C_{j}}{2}$ for $j=3, \ldots, d$ and $E_{j}=0$ for every $j$. Then, by Theorem 3.1, there exists an abelian cover $\pi: Z \rightarrow X$ ramified over $\mathcal{C}$ with ramification index 2. The Galois group $G$ of $\pi$ is generated by $v_{1}=v_{2}, v_{3}, \ldots, v_{d}$ and no proper subset of $\left\{v_{2}, \ldots, v_{d}\right\}$ generates $G$. Note that every element of $G$ has order 2. So the Galois group of $\pi$ is $(\mathbb{Z} / 2 \mathbb{Z})^{d-1}$. We denote by $\rho: Y \rightarrow Z$ the minimal desingularization of $Z$.

For a singular point $p$ of $\mathcal{C}$, recall that $r_{p}$ denotes its multiplicity. Let $\tau: \widetilde{X} \rightarrow X$ be the blow up of $X$ at the $f_{0}-t_{2}=\sum_{k \geq 3} t_{k}$ singular points of $\mathcal{C}$ with multiplicities $k \geq 3$. Let $\widetilde{D}=\sum_{i=1}^{d} \widetilde{C}_{i}$ be the strict transform of $D$ in $\widetilde{X}$ and let $E_{p}:=\tau^{-1}(p)$ be the exceptional divisor over the point $p$. 
Note that the singular locus of $Z$ is precisely the pre-image, under $\pi$, of the singular points of $\mathcal{C}$ of multiplicity at least 3 (see [17, Proposition 3.1], for example). Since $\tau$ is defined to be the blow up of the singular points of $\mathcal{C}$ of multiplicity at least 3, there exists a morphism $\sigma: Y \rightarrow \widetilde{X}$, by the universal property of blow ups. See the commutative diagram in Figure 1.

From the commutativity of the diagram, it is easy to see that $\sigma$ is also an abelian cover with Galois group $(\mathbb{Z} / 2 \mathbb{Z})^{d-1}$, branch divisor $\widetilde{D}$ and ramification index 2 at every irreducible component of $\widetilde{D}$. Then $\sigma^{\star} E_{p}$ is a divisor in $Y$ consisting of $2^{d-1-r_{p}}$ disjoint curves $F_{p}$, each with multiplicity 2. See [9, II.3.2] for more details. For a point $x \in E_{p}$ which is not in the branch locus of $\sigma, \sigma^{-1}(x)$ consists of $2^{d-1}$ distinct points and these are contained in the $2^{d-1-r_{p}}$ disjoint curves $F_{p}$. Since each $F_{p}$ occurs with multiplicity 2 in $\sigma^{\star} E_{p}$, the number of elements in a single $F_{p}$ that map to $x$ is $\frac{2^{d-1}}{2\left(2^{\left.d-1-r_{p}\right)}\right.}=2^{r_{p}-1}$. So each $F_{p}$ is a finite cover of $E_{p}$ of degree $2^{r_{p}-1}$. The branch locus of the map $F_{p} \rightarrow E_{p}$ is precisely the $r_{p}$ intersection points of $E_{p}$ and $\widetilde{D}$. Since the ramification index is 2 and the degree of the map $F_{p} \rightarrow E_{p}$ is $2^{r_{p}-1}$, there are $\frac{2^{r_{p}-1}}{2}=2^{r_{p}-2}$ points in $F_{p}$ that map to any point in the branch locus. Hence the degree of the ramification divisor is $2^{r_{p}-2} r_{p}$.

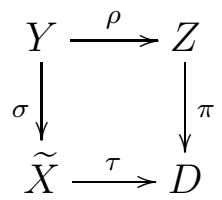

FiguRE 1. Construction of the surface $Y$

By the above discussion, we have $\sigma^{\star} E_{p}=\sum 2 F_{p}$ with $2^{d-1-r_{p}}$ terms in the summand. So

$$
-2^{d-1}=2^{d-1}\left(E_{p}\right)^{2}=\left(\sigma^{\star} E_{p}\right)^{2}=4\left(2^{d-1-r_{p}}\right) F_{p}^{2},
$$

which implies that $F_{p}^{2}=-2^{r_{p}-2}$ for every point $p \in \operatorname{Sing}(\mathcal{C})$ with $r_{p} \geq 3$.

Using the Hurwitz formula to compute the Euler characteristic of $F_{p}$, we get

$$
e\left(F_{p}\right)=2-2 g\left(F_{p}\right)=2^{r_{p}-1}(2)-2^{r_{p}-2} r_{p}=2^{r_{p}-2}\left(4-r_{p}\right) .
$$

We will calculate the Chern numbers $c_{2}, c_{1}^{2}$ of $Y$, where $c_{2}$ is same as the Euler characteristic $e(Y)$ of $Y$ and $c_{1}^{2}$ is the self-intersection number of a canonical divisor of $Y$.

Note that

$$
Y \backslash \bigcup_{p, r_{p} \geq 3} \sigma^{-1} E_{p}=(\tau \circ \sigma)^{-1}\left((X \backslash \mathcal{C}) \cup(\mathcal{C} \backslash \operatorname{Sing}(\mathcal{C})) \cup\left\{p \in \operatorname{Sing}(\mathcal{C}) \mid r_{p}=2\right\}\right) .
$$

If $A \rightarrow B$ is an étale map of degree $n$, then $e(A)=n e(B)$. Since $\sigma$ is an étale map on $Y \backslash \bigcup_{p, r_{p} \geq 3} \sigma^{-1} E_{p}$, we get

$$
e\left(Y \backslash \bigcup_{p, r_{p} \geq 3} \sigma^{-1} E_{p}\right)=2^{d-1} e(X \backslash \mathcal{C})+2^{d-2} e(\mathcal{C} \backslash \operatorname{Sing}(\mathcal{C}))+2^{d-3} t_{2}
$$


Using the additivity of the topological Euler characteristic, we have the following:

$$
\begin{aligned}
& e(\mathcal{C})=2 \sum\left(1-g\left(C_{i}\right)\right)-\sum_{k \geq 2}(k-1) t_{k}, \\
& e(\mathcal{C} \backslash \operatorname{Sing}(\mathcal{C}))=2 \sum\left(1-g\left(C_{i}\right)\right)-\sum_{k \geq 2} k t_{k}, \\
& e(X \backslash \mathcal{C})=e(X)+2 \sum\left(g\left(C_{i}\right)-1\right)+\sum_{k \geq 2}(k-1) t_{k} .
\end{aligned}
$$

Substituting these values in (3.2), we have

$$
\begin{aligned}
e\left(Y \backslash \bigcup_{p, r_{p} \geq 3} \sigma^{-1} E_{p}\right)= & 2^{d-1}\left(e(X)+2 \sum\left(g\left(C_{i}\right)-1\right)+\sum_{k \geq 2}(k-1) t_{k}\right)+ \\
& 2^{d-2}\left(-2 \sum\left(g\left(C_{i}\right)-1\right)-\sum_{k \geq 2} k t_{k}\right)+2^{d-3} t_{2} .
\end{aligned}
$$

It is easy to check that

$$
e(X)=4-4 g \text { and } 2 g\left(C_{i}\right)-2=-a^{2} e+2 a b+a e+a(2 g-2)-2 b .
$$

Note also that $\sum_{k \geq 2}(k-1) t_{k}=f_{1}-f_{0}$.

So we get

$$
\begin{aligned}
e\left(Y \backslash \bigcup_{p, r_{p} \geq 3} \sigma^{-1} E_{p}\right)= & 2^{d-1}\left(4-4 g+d\left(-a^{2} e+2 a b+a e+a(2 g-2)-2 b\right)+f_{1}-f_{0}\right)+ \\
& 2^{d-2}\left(-d\left(-a^{2} e+2 a b+a e+a(2 g-2)-2 b\right)-f_{1}\right)+2^{d-3} t_{2} .
\end{aligned}
$$

There are $2^{d-1-r_{p}}$ curves with Euler characteristic $e\left(F_{p}\right)$ in $Y$ over each exceptional divisor $E_{p}$ in $\tilde{X}$. So (3.1) gives

$$
\begin{aligned}
e(Y) & =e\left(Y \backslash \bigcup_{p, r_{p} \geq 3} \sigma^{-1} E_{p}\right)+\sum_{k \geq 3} 2^{d-1-k} t_{k} e\left(F_{p}\right) \\
& =e\left(Y \backslash \bigcup_{p, r_{p} \geq 3} \sigma^{-1} E_{p}\right)+\sum_{k \geq 3} 2^{d-1-k} t_{k}\left(2^{k-1}(2-k)+k 2^{k-2}\right) \\
& =e\left(Y \backslash \bigcup_{p, r_{p} \geq 3} \sigma^{-1} E_{p}\right)+2^{d-3} \sum_{k \geq 3} t_{k}(4-k) \\
& =e\left(\begin{array}{c}
Y \backslash \bigcup_{p, r_{p} \geq 3} \sigma^{-1} E_{p} \\
Y
\end{array}\right)+2^{d-3}\left(4 f_{0}-f_{1}-2 t_{2}\right)
\end{aligned}
$$


Now using the value of $e\left(Y \backslash \bigcup_{p, r_{p} \geq 3} \sigma^{-1} E_{p}\right)$ computed above and simplifying, we get

$$
\frac{1}{2^{d-3}} e(Y)=16-16 g+d\left(-2 a^{2} e+4 a b+2 a e+4 a g-4 a-4 b\right)+f_{1}-t_{2} .
$$

Next we calculate $c_{1}^{2}(Y)$.

For the divisor $D=\sum_{i=1}^{d} C_{i}$ on $X$, we know that $\tau^{\star} D-\sum_{\substack{p \in \operatorname{Sing}(\mathcal{C}), r_{p} \geq 3}} r_{p} E_{p}$ is the strict transform of $D$ in $\tilde{X}$. The divisors $\sigma^{\star}\left(\tau^{\star} D-\sum r_{p} E_{p}\right)$ and $\sigma^{\star} E_{p}$ of $Y\left(p \in \operatorname{Sing}(\mathcal{C}), r_{p} \geq 3\right)$ are divisible by 2. For a canonical divisor $K_{X}$ of $X, \tau^{\star} K_{X}+\sum E_{p}$ is a canonical divisor of $\tilde{X}$. Applying [1, Page 42, Lemma 17.1] to the ramified covering $\sigma: Y \rightarrow \widetilde{X}$, we get the following:

Lemma 3.2. Let $Y$ be the surface constructed in Figure 1. The canonical divisor of $Y$ is given by $K_{Y}=\sigma^{\star} T$ for the $\mathbb{Q}$-divisor $T$ on $\widetilde{X}$ defined as

$$
T:=\tau^{\star} K_{X}+\sum E_{p}+\frac{1}{2}\left(\sum E_{p}+\tau^{*} D-\sum r_{p} E_{p}\right),
$$

where the summations are taken over all the points $p \in \operatorname{Sing}(\mathcal{C})$ such that $r_{p} \geq 3$.

Thus, $T^{2}=K_{X}^{2}+K_{X} \cdot D-\sum_{k \geq 3} t_{k}+\sum_{k \geq 3}(k-1) t_{k}+\frac{1}{4}\left(D^{2}-\sum_{k \geq 3}(k-1)^{2} t_{k}\right)$.

We have the following:

$$
\begin{aligned}
& K_{X}^{2}=8(1-g) \\
& K_{X} \cdot D=d(a e+a(2 g-2)-2 b) \\
& \sum_{k \geq 3} t_{k}=f_{0}-t_{2}, \sum_{k \geq 3}(k-1) t_{k}=f_{1}-f_{0}-t_{2}, \text { and } \\
& D^{2}-\sum_{k \geq 3}(k-1)^{2} t_{k}=d\left(-a^{2} e+2 a b\right)+f_{1}-f_{0}+t_{2} . \text { For this equality, use Lemma } \\
& 2.5(2) .
\end{aligned}
$$

Substituting these values in the expression for $T^{2}$ and noting that $c_{1}^{2}(Y)=2^{d-1} T^{2}$, we get:

$$
\frac{1}{2^{d-3}} c_{1}^{2}(Y)=32-32 g+d\left(-a^{2} e+2 a b+4 a e+8 a g-8 a-8 b\right)-9 f_{0}+5 f_{1}+t_{2} .
$$

Now we have, by (3.3) and (3.4),

$$
\frac{1}{2^{d-3}}\left(3 e(Y)-c_{1}^{2}(Y)\right)=16-16 g+d[(2 b-a e)(5 a-2)+4 a(g-1)]+9 f_{0}-2 f_{1}-4 t_{2} .
$$

Remark 3.3. By (3.1), $F_{p}$ is rational if and only if $r_{p}=3$ and $F_{p}$ is elliptic if and only if $r_{p}=4$. Thus we know that $Y$ contains $2^{d-4} t_{3}$ disjoint $(-2)$-curves (above the 3 -points) and contains $2^{d-5} t_{4}$ elliptic curves (above the 4-points), each of self-intersection -4 .

\section{Harbourne Constants}

In this section, we will first show that the surface $Y$ (constructed in the last section; see Figure 1) has non-negative Kodaira dimension. This will allow us to apply a HirzebruchMiyaoka-Sakai inequality involving the Chern numbers of $Y$ and certain curves on $Y$ coming 
from the arrangement $\mathcal{C}$ on $X$ (see Theorem 4.6). Using this we obtain a Hirzebruch-type inequality (4.9). We prove our bound for the Harbourne constant of $\mathcal{C}$ in Theorem 4.7.

We will use the notation of Section 3, Recall that $T$ is a $\mathbb{Q}$-divisor on $\widetilde{X}$ defined in Lemma 3.2. We start with the following.

Lemma 4.1. Let $X$ be a ruled surface with $e \geq 4$. Let $\mathcal{C}$ be a transversal arrangement of curves satisfying Assumption 2.4. Then $T \cdot E_{p} \geq 0$ for every $p \in \operatorname{Sing}(\mathcal{C})$ such that $r_{p} \geq 3$.

Proof. T. $E_{p}=-1+\frac{-1+r_{p}}{2} \geq-1+\frac{-1+3}{2}=0$.

Lemma 4.2. Let $X$ be a ruled surface with $e \geq 4$. Let $\mathcal{C}$ be a transversal arrangement of curves satisfying Assumption 2.4. Let $C_{j}^{\prime}=\tau^{\star} C_{j}-\sum_{p \in C_{j}, r_{p} \geq 3} E_{p}$ be the strict transform of $C_{j} \in \mathcal{C}$, for $j=1,2, \ldots, d$. Then $T \cdot C_{j}^{\prime} \geq 0$.

Proof. Let $f_{0}^{j}$ denote the number of multiple points on $C_{j}$ and let $t_{k}^{j}$ denote the number of $k$-fold points on $C_{j}$.

Now,

$$
T \cdot C_{j}^{\prime}=K_{X} \cdot C_{j}+\frac{D \cdot C_{j}}{2}-T \cdot \sum_{p \in C_{j}, r_{p} \geq 3} E_{p} .
$$

We now compute each of the terms individually.

$$
\begin{aligned}
K_{X} \cdot C_{j} & =2 a e-2 b+(2 g-2-e) a, \\
D \cdot C_{j} & =d\left(2 a b-a^{2} e\right), \\
T \cdot E_{p} & =\frac{r_{p}-3}{2} ; \quad p \in C_{j}, r_{p} \geq 3 .
\end{aligned}
$$

By Lemma 2.5 (1), we have

$$
\begin{aligned}
T \cdot \sum_{p \in C_{j}, r_{p} \geq 3} E_{p} & =\sum_{p \in C_{j}, r_{p} \geq 3} \frac{r_{p}-3}{2} \\
& =\sum_{p \in C_{j}, r_{p} \geq 2} \frac{r_{p}-1}{2}-f_{0}^{j}+\frac{t_{2}^{j}}{2} \\
& =\frac{\left(2 a b-a^{2} e\right)(d-1)}{2}-f_{0}^{j}+\frac{t_{2}^{j}}{2} .
\end{aligned}
$$

Plugging the values computed above in (4.1), we get

$$
T \cdot C_{j}^{\prime}=2 a e-2 b+(2 g-e-2) a+\frac{2 a b-a^{2} e}{2}+f_{0}^{j}-\frac{t_{2}^{j}}{2} .
$$

To prove the lemma, it suffices to show

$$
f_{0}^{j}-\frac{t_{2}^{j}}{2} \geq-\left(\frac{2 a b-a^{2} e}{2}\right)-2 a e+a(e+2)+2 b .
$$


Let $k$ be the maximum of the multiplicities of the points on $C_{j}$. By Lemma 2.5 (1), we have

$$
t_{2}^{j}+2 t_{3}^{j}+\ldots+(k-1) t_{k}^{j}=\left(2 a b-a^{2} e\right)(d-1) .
$$

Now,

$$
\begin{aligned}
f_{0}^{j}-\frac{t_{2}^{j}}{2} & =\frac{t_{2}^{j}}{2}+t_{3}^{j}+\ldots+t_{k}^{j} \\
& \geq \frac{t_{2}^{j}+2 t_{3}^{j}+\ldots+(k-1) t_{k}^{j}}{k}=\frac{\left(2 a b-a^{2} e\right)(d-1)}{k} \\
& \geq 2 a b-a^{2} e
\end{aligned}
$$

where last inequality holds since $k \leq d-1$.

Thus in order to show (4.3), it suffices to show the following inequality:

$$
2 a b-a^{2} e \geq-\left(\frac{2 a b-a^{2} e}{2}\right)-2 a e+a(e+2)+2 b .
$$

Now we have the following:

$$
\begin{aligned}
\text { (4.4) } & \Leftrightarrow 6 a b-4 a-4 b \geq 3 a^{2} e-2 a e \\
& \Leftarrow b \geq \frac{4 a}{3 a-2} \\
& \Leftarrow a e \geq \frac{4 a}{3 a-2} \\
& \Leftarrow e \geq 4 .
\end{aligned}
$$

The last inequality holds by Assumption 2.4.

We now make a further assumption on our arrangement $\mathcal{C}$. This is required for our argument showing that $K_{Y}$ is nef.

Assumption 4.3. Let $X$ be a ruled surface over a smooth curve with $e \geq 4$. Let $\mathcal{C}$ be a transversal arrangement of curves on a ruled surface $X$ satisfying Assumption 2.4. Assume further that $\mathcal{C}$ satisfies one of the following conditions:

(1) $a \geq 2$, or

(2) $a=1$ and there exists a subset of four curves in $\mathcal{C}$ such that there is no point common to all the four curves.

Question 4.4. We do not know any example of a transversal arrangement for which Assumption 4.3 does not hold. Does this assumption always hold for any arrangement satisfying Assumption 2.4?

Theorem 4.5. Let $X$ be a ruled surface with $e \geq 4$ and let $\mathcal{C}$ be a transversal arrangement of curves satisfying Assumption 4.3. Let $Y$ be the surface constructed in Figure 1. Then $K_{Y}$ is nef. 
Proof. Recall (see Lemma 3.2) that $T$ is a divisor on $\widetilde{X}$ given by

$$
T:=\tau^{\star} K_{X}+\frac{3}{2} \sum_{r_{p} \geq 3} E_{p}+\frac{1}{2} \sum C_{i}^{\prime}
$$

where $C_{i}^{\prime}$ is the strict transform of $C_{i}$ by $\tau$ and $E_{p}=\tau^{-1}(p)$. Note that $K_{Y}=\sigma^{\star} T$. We have $\tau^{\star} C_{i}=C_{i}^{\prime}+\sum_{p \in C_{i}, r_{p} \geq 3} E_{p}$

We want to express $T$ as a positive sum of effective divisors on $\widetilde{X}$. The negative terms in the expression occur because of the term involving $K_{X}=-2 C_{0}+(2 g-2-e) f$. We consider two different cases.

Case (1): Assume $a \geq 2$. Let $C_{1}, C_{2} \in \mathcal{C}$.

For $q:=a-2 \geq 0, p:=2 g-e-2+b \geq 0$, we have $K_{X}=p f+q C_{0}-\frac{C_{1}+C_{2}}{2}$. Note that $p>0$, since $b \geq a e$ and $e \geq 4$.

Thus, (4.5) becomes,

$$
\begin{aligned}
T & =\tau^{\star}\left(p f+q C_{0}\right)-\frac{1}{2}\left(C_{1}^{\prime}+\sum_{p \in C_{1}, r_{p} \geq 3} E_{p}+C_{2}^{\prime}+\sum_{p \in C_{2}, r_{p} \geq 3} E_{p}\right)+\frac{3}{2} \sum_{r_{p} \geq 3} E_{p}+\frac{1}{2} \sum_{i=1}^{d} C_{i}^{\prime} \\
& =\tau^{\star}\left(p f+q C_{0}\right)+\frac{1}{2} \sum_{i=3}^{d} C_{i}^{\prime}+\sum \lambda_{p} E_{p}, \text { for some } \lambda_{p} .
\end{aligned}
$$

Note that $\lambda_{p}$ is non-negative for every point $p \in \operatorname{Sing}(\mathcal{C})$ with $r_{p} \geq 3$. Indeed, $\lambda_{p}=\frac{3}{2}$ if $p \notin C_{1} \cup C_{2} ; \lambda_{p}=1$ if $p$ belongs to exactly one of the curves $C_{1}$ or $C_{2}$; and $\lambda_{p}=\frac{1}{2}$ if $p \in C_{1} \cap C_{2}$. Thus $T$ is effective and we have

$$
K_{Y}=\sigma^{\star} T=\sigma^{\star} \tau^{\star}\left(p f+q C_{0}\right)+\sigma^{\star}\left(\frac{1}{2} \sum_{i=3}^{d} C_{i}^{\prime}\right)+\sigma^{\star}\left(\sum \lambda_{p} E_{p}\right) .
$$

If $C$ is a curve in $Y$ not contained in $\sigma^{\star} E_{p}$ and $\sigma^{\star} C_{i}^{\prime}$,

$$
K_{Y} \cdot C=0+\sigma^{\star}\left(\frac{1}{2} \sum_{i=3}^{d} C_{i}^{\prime}\right) \cdot C+\sigma^{\star}\left(\sum \lambda_{p} E_{p}\right) \cdot C \geq 0
$$

If $C$ is a curve in $Y$ such that $C$ is either $\sigma^{\star} C_{i}^{\prime}$ or in $\sigma^{\star} E_{p}$, Lemma 4.1 and Lemma 4.2 imply that $K_{Y} \cdot C \geq 0$. Thus $K_{Y} \cdot C \geq 0$ for every curve $C$ in $Y$. Hence, $K_{Y}$ is nef.

Case (2): Suppose that $a=1$. By Assumption 4.3, there are four curves, say $C_{1}, C_{2}, C_{3}, C_{4}$, in $\mathcal{C}$ such that no point is contained in all the four curves.

Let $p:=2 g-2-e+2 b>0$. Then $K_{X}=p f-\frac{C_{1}+C_{2}+C_{3}+C_{4}}{2}$. 
Thus,

$$
\begin{aligned}
T & =\tau^{\star}(p f)-\frac{1}{2}\left(\sum_{i=1}^{4} C_{i}^{\prime}+\sum_{p \in C_{i}, r_{p} \geq 3} E_{p}\right)+\frac{3}{2} \sum_{r_{p} \geq 3} E_{p}+\frac{1}{2} \sum_{i=1}^{d} C_{i}^{\prime} . \\
& =\tau^{\star}(p f)-\frac{1}{2}\left(\sum_{p \in C_{i}, r_{p} \geq 3} E_{p}\right)+\frac{3}{2} \sum_{r_{p} \geq 3} E_{p}+\frac{1}{2} \sum_{i=5}^{d} C_{i}^{\prime} . \\
& =\tau^{\star}(p f)+\frac{1}{2} \sum_{i=5}^{d} C_{i}^{\prime}+\sum \lambda_{p}^{\prime} E_{p}, \text { for some } \lambda_{p}^{\prime} .
\end{aligned}
$$

We have $\lambda_{p}^{\prime}=\frac{3}{2}$ if $p \notin C_{1} \cup C_{2} \cup C_{3} \cup C_{4}$. By Assumption 4.3 and the choice of $C_{1}, C_{2}, C_{3}, C_{4}$, there are no points in the intersection $C_{1} \cap C_{2} \cap C_{3} \cap C_{4}$. If $p$ belongs to three of them, then $\lambda_{p}^{\prime}=\frac{3}{2}-\frac{3}{2}=0$. So we have $\lambda_{p}^{\prime} \geq 0$ for all $p \in \operatorname{Sing}(\mathcal{C})$ with $r_{p} \geq 3$. Thus $T$ is effective and we have

$$
K_{Y}=\sigma^{\star} \tau^{\star}(p f)+\frac{1}{2} \sigma^{\star}\left(\sum_{i=5}^{d} C_{i}^{\prime}\right)+\sigma^{\star}\left(\sum \lambda_{p}^{\prime} E_{p}\right) .
$$

If $C$ is a curve in $Y$ not contained in $\sigma^{\star} E_{p}$ and $\sigma^{\star} C_{i}^{\prime}$,

$$
K_{Y} \cdot C=0+\sigma^{\star}\left(\frac{1}{2} \sum_{i=5}^{d} C_{i}^{\prime}\right) \cdot C+\sigma^{\star}\left(\sum \lambda_{p}^{\prime} E_{p}\right) \cdot C \geq 0 .
$$

If $C$ is a curve in $Y$ such that $C$ is either $\sigma^{\star} C_{i}^{\prime}$ or in $\sigma^{\star} E_{p}$, Lemma 4.1 and Lemma 4.2 imply that $K_{Y} \cdot C \geq 0$. Thus $K_{Y} \cdot C \geq 0$ for every curve $C$ in $Y$. Hence, $K_{Y}$ is nef.

The following result of Hirzebruch [12, Theorem 3, Page 144] is crucial in our computations. It strengthens earlier results of Miyaoka and Sakai.

Theorem 4.6 (Hirzebruch). Let $X$ be a smooth surface of general type and $E_{1}, \ldots, E_{k}$ configurations (disjoint to each other) of rational curves on $X$ (arising from quotient singularities) and $C_{1}, \ldots, C_{p}$ smooth elliptic curves (disjoint to each other and disjoint to the $E_{i}$ ). Let $c_{1}^{2}(X), c_{2}(X)$ be the Chern numbers of $X$. Then

$$
3 c_{2}(X)-c_{1}^{2}(X) \geq \sum_{j=1}^{p}\left(-C_{j}^{2}\right)+\sum_{i=1}^{k} m\left(E_{i}\right) .
$$

Hirzebruch in fact remarks that the result also holds when $X$ has non-negative Kodaira dimension. We use the theorem in this case.

The numbers $m\left(E_{i}\right)$ mentioned in the theorem are positive numbers defined using certain invariants (Euler characteristics, self-intersections) of the arrangements $E_{i}$. Hirzebruch gives a formula to compute them in [12, Page 144, (5)] which shows that if $E_{i}$ is a single (-2)-curve, then $m\left(E_{i}\right)=\frac{9}{2}$. See also [8].

Now we are ready to prove the main result of this paper. 
Theorem 4.7. Let $X$ be a ruled surface with $e \geq 4$ over a smooth curve of genus $g$. Let $\mathcal{C}$ be a transversal arrangement of curves satisfying Assumption 4.3. In particular, each curve in $\mathcal{C}$ is numerically equivalent to $a C_{0}+b f$ with $a>0$ and $b \geq a e$. Then we have the following bound on the Harbourne constant of $\mathcal{C}$ :

$$
H(X, \mathcal{C}) \geq \frac{-9}{2}-\frac{8}{f_{0}}+\frac{d}{f_{0}}\left(\frac{(a e-2 b)}{2}(3 a-2)-2 a(g-1)\right)+\frac{16 g+4 t_{2}+t_{4}}{2 f_{0}}+\frac{9 t_{3}}{8 f_{0}}
$$

Proof. By Remark 3.3, the surface $Y$ (constructed in Figure 1) contains $2^{d-4} t_{3}$ disjoint rational (-2)-curves $E_{i}$ (above the 3 -points) and contains $2^{d-5} t_{4}$ elliptic curves $C_{j}$ (above the 4-points), each of self-intersection -4 .

By Theorem 4.5, $K_{Y}$ is nef. Thus, by Theorem 4.6:

$$
\frac{3 c_{2}(Y)-c_{1}^{2}(Y)}{2^{d-3}} \geq \frac{\sum\left(-C_{j}^{2}\right)+\sum m\left(E_{i}\right)}{2^{d-3}} .
$$

As noted earlier, $m\left(E_{i}\right)=\frac{9}{2}$ for all rational curves $E_{i}$ of self-intersection -2 .

From (3.5), we have,

$$
\frac{1}{2^{d-3}}\left(3 e(Y)-c_{1}^{2}(Y)\right)=16-16 g+d[(2 b-a e)(5 a-2)+4 a(g-1)]+9 f_{0}-2 f_{1}-4 t_{2} .
$$

Also, from our discussion above,we have

$$
\begin{aligned}
\sum m\left(E_{i}\right) & =\frac{9}{2} 2^{d-4} t_{3}, \text { and } \\
\sum\left(-C_{j}^{2}\right) & =4 t_{4} 2^{d-5} .
\end{aligned}
$$

Plugging these values in (4.7) and simplifying, we have :

$$
16-16 g+d\left(2 a e-5 a^{2} e+10 a b+4 a g-4 a-4 b\right)+9 f_{0}-2 f_{1}-4 t_{2}-t_{4}-\frac{9}{4} t_{3} \geq 0 .
$$

Simplifying and re-arranging (4.8), we obtain the following Hirzebruch-type inequality for $\mathcal{C}$ :

$$
t_{2}+\frac{3}{4} t_{3} \geq-16+16 g+\sum_{k \geq 5}(2 k-9) t_{k}+d\left(e\left(5 a^{2}-2 a\right)-10 a b-4 a g+4 a+4 b\right) .
$$

Now we bound $H(X, \mathcal{C})$. We have

$$
H(X, \mathcal{C})=\frac{\left(2 a b-a^{2} e\right) d^{2}-\sum_{k \geq 2} k^{2} t_{k}}{f_{0}}=\frac{\left(2 a b-a^{2} e\right) d^{2}-f_{2}}{f_{0}}=\frac{\left(2 a b-a^{2} e\right) d-f_{1}}{f_{0}},
$$

where the last equality follows from Lemma 2.5(2).

From (4.8), we have

$$
-f_{1} \geq \frac{-16+16 g+d\left(e\left(5 a^{2}-2 a\right)-10 a b-4 a g+4 a+4 b\right)-9 f_{0}+4 t_{2}+\frac{9}{4} t_{3}+t_{4}}{2} .
$$


Thus,

$$
\begin{aligned}
H(X, \mathcal{C}) & \geq \frac{d\left(-a^{2} e+2 a b\right)-8+8 g+\frac{d\left(e\left(5 a^{2}-2 a\right)-10 a b-4 a g+4 a+4 b\right)-9 f_{0}}{2}+2 t_{2}+\frac{9}{8} t_{3}+\frac{t_{4}}{2}}{f_{0}} \\
& =\frac{-9}{2}-\frac{8}{f_{0}}+\frac{d}{f_{0}}\left(\frac{a e}{2}(3 a-2)-2 a g-3 a b+2 a+2 b\right)+\frac{16 g+4 t_{2}+t_{4}}{2 f_{0}}+\frac{9 t_{3}}{8 f_{0}} .
\end{aligned}
$$

This completes the proof of the theorem.

If the curves in the arrangement $\mathcal{C}$ do not intersect the normalized section $C_{0}$, then we obtain an improved bound for the $H$-constants as shown in the following proposition. We obtain an improved bound in this case because $Y$ contains some additional rational curves.

Proposition 4.8. Let $X$ be a ruled surface with $e \geq 4$ over a smooth curve of genus $g$. Let $\mathcal{C}$ be a transversal arrangement of curves satisfying Assumption 4.3. Assume further that no curve in $\mathcal{C}$ intersects the normalized section $C_{0}$. Then we have the following bound on the Harbourne constant of $\mathcal{C}$ :

$$
H(X, \mathcal{C}) \geq \frac{-9}{2}+\frac{d}{f_{0}}\left(\frac{a e(2-3 a)-4 a(g-1)}{2}\right)+\frac{16 g+4 t_{2}+t_{4}}{2 f_{0}}+\frac{9 t_{3}}{8 f_{0}}
$$

Proof. As in the previous theorem, by Remark 3.3, the surface $Y$ contains $2^{d-4} t_{3}$ disjoint rational (-2)-curves $E_{i}$ (above the 3 -points), $2^{d-5} t_{4}$ elliptic curves $C_{j}$ (above the 4 -points), each of self-intersection -4 . Further, since the curves in the arrangement do not intersect $C_{0}$, the surface $\tilde{X}$ has an isomorphic copy of $C_{0}$. Hence $Y$ contains $2^{d-1}$ copies of a rational curve $H$ of self-intersection $-e$.

Hirzebruch gives a formula to compute the value $m(H)$ in [12, Page 144, (4)]. Applying this formula, we have that for rational curves $H$ of self-intersection $-e, m(H)=2+e+\frac{1}{e}$.

By Theorem 4.5, $K_{Y}$ is nef. Thus, by Theorem 4.6, the inequality in (4.7) is satisfied.

From (3.5), we have,

$$
\frac{1}{2^{d-3}}\left(3 e(Y)-c_{1}^{2}(Y)\right)=16-16 g+d[(2 b-a e)(5 a-2)+4 a(g-1)]+9 f_{0}-2 f_{1}-4 t_{2} .
$$

We have

$$
\begin{aligned}
\sum m\left(E_{i}\right)+\sum m(H) & =\frac{9}{2} 2^{d-4} t_{3}+2^{d-1}\left(2+e+\frac{1}{e}\right), \text { and } \\
\sum\left(-C_{j}^{2}\right) & =4 t_{4} 2^{d-5} .
\end{aligned}
$$

Plugging these values in (4.7) and simplifying, we have:

$$
16-16 g+d\left(2 a e-5 a^{2} e+10 a b+4 a g-4 a-4 b\right)+9 f_{0}-2 f_{1}-4 t_{2}-t_{4}-\frac{9}{4} t_{3}-4\left(2+e+\frac{1}{e}\right) \geq 0 .
$$

Simplifying (4.11), with $a e=b$, we arrive at the following modified Hirzebruch-type inequality for $\mathcal{C}$ :

$$
t_{2}+\frac{3}{4} t_{3} \geq 4\left(e+\frac{1}{e}\right)-8+16 g+\sum_{k \geq 5}(2 k-9) t_{k}+d\left(-5 a^{2} e+2 a e-4 a g+4 a\right)
$$


Since $e \geq 4$, we have $4\left(e+\frac{1}{e}\right) \geq 17$. So (4.12) becomes:

$$
t_{2}+\frac{3}{4} t_{3} \geq 9+16 g+\sum_{k \geq 5}(2 k-9) t_{k}+d\left(-5 a^{2} e+2 a e-4 a g+4 a\right) \text {. }
$$

From the above inequality (4.13), we have

$$
-f_{1} \geq \frac{9+16 g+d\left(e\left(2 a-5 a^{2}\right)-4 a g+4 a\right)-9 f_{0}+4 t_{2}+\frac{9}{4} t_{3}+t_{4}}{2} .
$$

We now bound the $H$-constant $H(X, \mathcal{C})$.

$$
\begin{aligned}
H(X, \mathcal{C}) & \geq \frac{d\left(-a^{2} e+2 a b\right)+8 g+\frac{d\left(e\left(2 a-5 a^{2}\right)-4 a g+4 a\right)-9 f_{0}+9}{2}+2 t_{2}+\frac{9}{8} t_{3}+\frac{t_{4}}{2}}{f_{0}} \\
& \geq \frac{-9}{2}+\frac{d}{f_{0}}\left(\frac{-7 a^{2} e}{2}+2 a b+a e-2 a g+2 a\right)+\frac{16 g+4 t_{2}+t_{4}}{2 f_{0}}+\frac{9 t_{3}}{8 f_{0}} .
\end{aligned}
$$

Since $a e=b$, we get

$$
H(X, \mathcal{C}) \geq \frac{-9}{2}+\frac{d}{f_{0}}\left(\frac{a e(2-3 a)-4 a(g-1)}{2}\right)+\frac{16 g+4 t_{2}+t_{4}}{2 f_{0}}+\frac{9 t_{3}}{8 f_{0}},
$$

as required.

We now define the $H$-constant of a ruled surface for a fixed pair of integers $a, b$ as follows.

Definition 4.9. Let $X$ be a ruled surface with invariant $e \geq 4$. Let $a>0$ and $b \geq a e$ be positive integers. We define the $H$-constant $H_{a, b}(X)$ of $X$ as :

$$
H_{a, b}(X):=\inf _{\mathcal{C}} H(X, \mathcal{C})
$$

where the infimum is over all transversal arrangements $\mathcal{C}$ satisfying Assumption 4.3 ,

In order to bound the constant $H_{a, b}(X)$, we make the following observation.

Lemma 4.10. Let $\mathcal{C}=\left\{C_{1}, C_{2}, \ldots, C_{d}\right\}$ be a transversal arrangement on the ruled surface $X$ satisfying Assumption 4.3. Then $f_{0} \geq d$.

Proof. This is proved in [6, Lemma 6.1]. We write the proof here for the convenience of the reader.

Let $s=f_{0}$ and $h=2 a b-a^{2} e$. Let $\operatorname{Sing}(\mathcal{C})=\left\{p_{1}, \ldots, p_{s}\right\}$. Consider the $\mathbb{Q}$-vector space $\mathbb{Q}^{s}$ with the usual dot product: if $v=\left(a_{1}, \ldots, a_{s}\right)$ and $u=\left(b_{1}, \ldots, b_{s}\right)$, then $v \cdot u:=$ $a_{1} b_{1}+\ldots+a_{s} b_{s}$.

For every curve $C_{i} \in \mathcal{C}$, we associate a vector $v_{i} \in \mathbb{Q}^{s}$ by setting the $l$-th entry of $v_{i}$ equal to 1 , if $C_{i}$ passes through $p_{l}$, and 0 otherwise.

Note that if $i \neq j$, then $v_{i} \cdot v_{j}$ is precisely the number of points common to $C_{i}$ and $C_{j}$. By our hypothesis, we have $v_{i} \cdot v_{j}=h$. Also $v_{i} \cdot v_{i}$ is the number of multiple points that are contained in $C_{i}$. 
We claim that each curve $C_{i}$ contains at least $h+1$ intersection points with other curves in the arrangement. Since there are at least two curves in $\mathcal{C}$, we have $v_{i} \cdot v_{i} \geq h$. If $v_{i} \cdot v_{i}=h$, then all the curves in $\mathcal{C}$ intersect $C_{i}$ in the same $h$ points. This contradicts the assumption $t_{d}=0$. Thus $v_{i} \cdot v_{i}>h$ for all $i$.

To prove the lemma, it suffices to show that the set $\left\{v_{1}, v_{2}, \ldots, v_{d}\right\}$ is linearly independent. If it is not linearly independent, without loss of generality, let $v_{1}=\sum_{j=2}^{d} a_{j} v_{j}$ for $a_{j} \in \mathbb{Q}$.

Consider $v_{1} \cdot\left(v_{1}-v_{q}\right)$ where $q \geq 2$. Then

$$
\begin{aligned}
\left(v_{1} \cdot v_{1}\right)-h & =v_{1} \cdot\left(v_{1}-v_{q}\right) \\
& =\left(\sum_{j=2}^{d} a_{j} v_{j}\right) \cdot\left(v_{1}-v_{q}\right) \\
& =\sum_{j=2}^{d} a_{j}\left(h-\left(v_{j} \cdot v_{q}\right)\right) \\
& =a_{q}\left(h-\left(v_{q} \cdot v_{q}\right)\right)
\end{aligned}
$$

So $a_{q}=\frac{\left(v_{1} \cdot v_{1}\right)-h}{h-\left(v_{q} \cdot v_{q}\right)}<0$. Since this holds for all $q \geq 2, v_{1}$ is a linear combination of $v_{2}, \ldots, v_{d}$ with negative coefficients. But the entries of $v_{i}$ for any $i=1, \ldots, d$ are either 0 or 1 and we obtain the required contradiction.

Corollary 4.11. Let $X$ be a ruled surface over a smooth curve of genus $g$ with invariant $e \geq 4$. Let $a>0$ and $b>$ ae be positive integers. Then

$$
H_{a, b}(X) \geq \frac{-11}{2}+\frac{(a e-2 b)}{2}(3 a-2)-2 a g .
$$

Further, if $a e=b$, then

$$
H_{a, b}(X) \geq \frac{-9}{2}+\frac{a e(2-3 a)-4 a g}{2}
$$

Proof. We first claim that $f_{0}>2 a b-a^{2} e+1$. Indeed, if not, $f_{0} \leq 2 a b-a^{2} e+1$. Then

$$
\begin{aligned}
\left(2 a b-a^{2} e\right) d(d-1) & =\sum_{k \geq 2} k(k-1) t_{k}, \text { by Lemma 2.5) }(2) \\
& \leq(d-1)(d-2) f_{0}, \text { since } k \leq d-1 \\
& \leq(d-1)(d-2)\left(2 a b-a^{2} e+1\right) .
\end{aligned}
$$

This gives

$$
\begin{aligned}
\left(2 a b-a^{2} e\right) d & \leq(d-2)\left(2 a b-a^{2} e+1\right) \\
\Rightarrow 2\left(2 a b-a^{2} e\right) & \leq(d-2) \\
\Rightarrow 2(d-1) & \leq 2\left(2 a b-a^{2} e\right) \leq(d-2), \text { by Lemma } 4.10 \\
\Rightarrow d & \leq 0 .
\end{aligned}
$$

This is a contradiction and the claim follows. 
Now, since $b>a e, e \geq 4$ and $a>0$ by our assumptions, the claim gives $f_{0} \geq 2 a b-a^{2} e+2 \geq$ $2 a(a e+1)-a^{2} e+2 \geq 4 a^{2}+2 a+2 \geq 8$. Thus $f_{0} \geq 8$ and hence $\frac{-8}{f_{0}} \geq-1$.

By Theorem 4.7, we have $H(X, \mathcal{C}) \geq \frac{-9}{2}-\frac{8}{f_{0}}+\frac{d}{f_{0}}\left(\frac{(a e-2 b)}{2}(3 a-2)-2 a(g-1)\right)$. Note that $\frac{(a e-2 b)}{2}(3 a-2)-2 a g$ is a negative number as $b>a e$. Hence, as $\frac{-8}{f_{0}} \geq-1$, Lemma 4.10 gives (4.14).

Similarly, by Proposition 4.8, we have $H(X, \mathcal{C}) \geq \frac{-9}{2}+\frac{d}{f_{0}}\left(\frac{a e(2-3 a)-4 a(g-1)}{2}\right)$. Since $\frac{a e(2-3 a)-4 a g}{2}$ is a negative number, Lemma 4.10 gives (4.15).

We now state a corollary which gives a lower bound on the self-intersection of the strict transform of the divisor associated to an arrangement of curves.

Corollary 4.12. Let $\mathcal{C}$ be a transversal arrangement on the ruled surface $X$ satisfying Assumption 4.3. Let $f: \widetilde{X} \rightarrow X$ be the blow-up of $X$ at $\operatorname{Sing}(\mathcal{C})$. Let $\widetilde{D}$ denote the strict transform of $D$, which is the divisor defined as the sum of all the curves in $\mathcal{C}$. Then

$$
\widetilde{D}^{2} \geq-8-\frac{9}{2} s+d\left(\frac{(a e-2 b)}{2}(3 a-2)-2 a(g-1)\right)+8 g+2 t_{2}+\frac{t_{4}}{2}+\frac{9 t_{3}}{8} .
$$

Further, if all curves in the arrangement do not intersect the normalized section $C_{0}$, then

$$
\widetilde{D}^{2} \geq \frac{-9}{2} s+d\left(\frac{a e(2-3 a)-4 a(g-1)}{2}\right)+8 g+2 t_{2}+\frac{t_{4}}{2}+\frac{9 t_{3}}{8} .
$$

Proof. Indeed, note that $f_{0}=s$ and $\widetilde{D}^{2}=s H(X, \mathcal{C})$. The corollary now follows from (4.6) and (4.10).

4.1. Examples. It is not easy to construct arrangements which have small Harbourne constants. Most easy to construct examples of curve arrangements have much larger Harbourne constants than our bounds predict. For example, if $\mathcal{C}=\left\{C_{1}, \ldots, C_{d}\right\}$ is a general arrangement of curves on a ruled surface $X$ satisfying our assumptions, then it is easy to see that $H(X, \mathcal{C})=\frac{-2(d-2)}{d-1}$. Indeed, all singular points of $\mathcal{C}$ have multiplicity 2 and consequently, $t_{2}=\left(\begin{array}{l}d \\ 2\end{array}\right) C_{1}^{2}$ and $t_{k}=0$ for $k \geq 3$. Now an easy calculation gives $H(X, \mathcal{C})=\frac{-2(d-2)}{d-1}$. But this value is much larger than the bounds given by our main results Theorem 4.7 or Corollary 4.11 .

This situation is analogous to the case of line arrangements in $\mathbb{P}^{2}$. The best bound we have in this case is given in [3, Theorem 3.3] which proves that $H\left(\mathbb{P}^{2}, \mathcal{L}\right)>-4$ for all line arrangements $\mathcal{L}$. But for a general line arrangement or for many simple examples, the Harbourne constant is at least -2 . However, there do exist line arrangements in the plane which have small Harbourne constants. We can use these to obtain fairly small Harbourne constants for curve arrangements on ruled surfaces. We illustrate this with two examples below.

Example 4.13. Let $X=X_{e}$ be a rational ruled surface with invariant $e \geq 1$. Given a line arrangement in $\mathbb{P}^{2}$, one can obtain an arrangement of curves on $X_{e}$, following a construction outlined in [6. Example 15], where a specific finite morphism $X_{e} \rightarrow X_{1}$ of degree $e$ is described. 
Note that $X_{1}$ is isomorphic to a blow up of $\mathbb{P}^{2}$ at a point. So we can pull-back lines in $\mathbb{P}^{2}$ to $X_{e}$ which are in the class $(1, e)$. If $\mathcal{L}$ is a line arrangement of $d$ lines in the plane, its pull-back gives a curve arrangement $\mathcal{C}$ of $d$ curves in $X_{e}$.

To be more precise, suppose that $\mathcal{L}$ has $s$ singularities and $t_{k}$ denotes the number of singular points of $\mathcal{L}$ of multiplicity $k$. Then the singular points of $\mathcal{C}$ are precisely the preimages of singularities of $\mathcal{L}$. So $\mathcal{C}$ has es singular points and the number of singular points of multiplicity $k$ is $e_{k}$. Note that each curve in $\mathcal{C}$ is in the class $(1, e)$ and has self-intersection $e$. So the self-intersection of the divisor associated to $\mathcal{C}$ is $d^{2} e$.

Hence we have

$$
H(X, \mathcal{C})=\frac{d^{2} e-e \sum_{p \in \operatorname{Sing}(\mathcal{L})} r_{p}^{2}}{s e}=\frac{d^{2}-\sum_{p \in \operatorname{Sing}(\mathcal{L})} r_{p}^{2}}{s}=H\left(\mathbb{P}^{2}, \mathcal{L}\right) .
$$

We now assume $e \geq 4$. First we consider the Klein arrangement [13], denoted by $\mathcal{L}_{1}$. This arrangement consists of 21 lines with $t_{3}=28, t_{4}=21$ and $t_{k}=0$ for $k \neq 3,4$. It is easy to see that $H\left(\mathbb{P}^{2}, \mathcal{L}_{1}\right)=-3$. So if $\mathcal{C}_{1}$ is the curve arrangement in $X$ obtained from $\mathcal{L}_{1}$, then $H\left(X, \mathcal{C}_{1}\right)=-3$.

Now we calculate the bound given by Proposition 4.8. (Note that since $a e=b$, this bound is better than the one given by Theorem 4.7.) We have $d=21, f_{0}=49 e, a=1, b=e, g=$ $0, t_{2}=0, t_{3}=28 e, t_{4}=21 e$. So Proposition 4.8 gives

$$
H\left(X, \mathcal{C}_{1}\right) \geq \frac{-9}{2}+\frac{21}{49 e}\left(\frac{4-e}{2}\right)+\frac{21 e}{98 e}+\frac{9(28)}{8(49)}=\frac{42}{49 e}-3.857
$$

Next let $\mathcal{L}_{2}$ denote the Wiman configuration [25]. This arrangement consists of 45 lines with $t_{3}=120, t_{4}=45, t_{5}=36$ and $t_{k}=0$ for $k \neq 3,4,5$. It is easy to check that $H\left(X, \mathcal{C}_{2}\right)=$ -3.359 , where $\mathcal{C}_{2}$ is the arrangement of curves in $X$ given by $\mathcal{L}_{2}$.

As above, using Proposition 4.8, we obtain

$$
H\left(X, \mathcal{C}_{2}\right) \geq \frac{-9}{2}+\frac{45}{201 e}\left(\frac{4-e}{2}\right)+\frac{45 e}{402 e}+\frac{9(120)}{8(201)}=\frac{90}{201 e}-3.828 .
$$

\section{BALL QUOTIENTS}

Ball quotients are algebraic surfaces for which the universal cover is the 2-dimensional unit ball. Equivalently, ball quotients are minimal smooth complex projective surfaces $Y$ of general type satisfying equality in the Bogomolov-Miyaoka-Yau inequality. In other words, they are minimal smooth complex projective surfaces $Y$ such that $K_{Y}$ is nef and big and $K_{Y}^{2}=3 e(Y)$, where $K_{Y}$ denotes the canonical divisor and $e(Y)$ is the topological Euler characteristic. See [24] for more details on ball quotients.

Hirzebruch 12 gave examples of ball quotients using line arrangements in $\mathbb{P}^{2}$. To a line arrangement in $\mathbb{P}^{2}$, he associated a surface $Y$ (by first an abelian cover of $\mathbb{P}^{2}$ branched on that line arrangement and then taking a desingularization). He exhibited three specific line arrangements whose associated surfaces $Y$ are ball quotients. 
In this section, we show that the surfaces associated to transversal arrangements on ruled surfaces that we consider in this paper are not ball quotients. In order to do this, we use the theory of constantly branched covers developed in [2]. The crucial idea is the following. Let $Y$ be a ball quotient which arises from the abelian cover construction we used in Section 3 , Then if $E$ is a curve contained in the ramification divisor of $\sigma: Y \rightarrow \widetilde{X}$, then the relative proportionality of $E$ is zero. This is defined as $\operatorname{prop}(E):=2 E^{2}-e(E)$. For more details, see [2, Section 1.3]. See also [11] for a nice introduction. In the notation of [11], one says that $Y$ is a good covering of $\tilde{X}$ via $\sigma$.

The same method was used in [19] and [20] to study ball quotients.

Let $X$ be a ruled surface with $e \geq 4$. Let $\mathcal{C}=\left\{C_{1}, C_{2}, \ldots, C_{d}\right\}$ be a transversal arrangement of curves on the ruled surface $X$ satisfying Assumption 4.3. Let $Y$ be the associated surface constructed in Section 3, see Figure 1. By Theorem 4.5, $K_{Y}$ is nef and consequently, $Y$ is a minimal surface of non-negative Kodaira dimension. In fact, $Y$ is a surface of general type most of the time as the following remark shows.

Remark 5.1. Let $\mathcal{C}$ be a transversal arrangement on the ruled surface $X$ satisfying Assumption 4.3. Assume in addition that $a \geq 8$. By (3.4), we have

$$
K_{Y}^{2}=2^{d-3}\left(32+(8 a d-32) g+d(a(2 b-a e)+4 a(e-2)-8 b)+5 f_{1}-9 f_{0}+t_{2}\right) .
$$

Using $a \geq 8$ and Assumption 4.3, it is easy to see that $K_{Y}^{2}>0$. Thus $Y$ is a minimal surface of general type.

We define the Hirzebruch polynomial as

$$
H_{\mathcal{C}}(2):=\frac{1}{2^{d-3}}\left(3 e(Y)-c_{1}^{2}(Y)\right)
$$

Note that by equation (3.5), we have

$$
H_{\mathcal{C}}(2)=16-16 g+d((2 b-a e)(5 a-2)+4 a(g-1))+9 f_{0}-2 f_{1}-4 t_{2} .
$$

By Theorem 4.5, $H_{\mathcal{C}}(2) \geq 0$. If $Y$ is a ball quotient then $H_{\mathcal{C}}(2)=0$.

We now check whether there exists a transversal arrangement $\mathcal{C}$ on $X$ satisfying Assumption 4.3 such that the associated surface $Y$ is a ball quotient.

As noted above, the relative proportionality of curves contained in the ramification divisor of $\sigma$ is zero. There are two kinds of curves which are contained in the ramification divisor of $\sigma$. The first kind are the irreducible components $F_{p}$ of $\sigma^{\star} E_{p}$ for $p \in \operatorname{Sing}(\mathcal{C})$ with $r_{p} \geq 3$. Since $F_{p}^{2}=-2^{r_{p}-2}$, (3.1) gives $\operatorname{prop}\left(F_{p}\right)=2^{r_{p}-2}\left(r_{p}-6\right)$.

So, if the associated surface $Y$ is a ball quotient, then for any point $p \in \operatorname{Sing}(\mathcal{C})$ with $r_{p} \geq 3$, we have $r_{p}=6$. Hence the arrangement $\mathcal{C}$ satisfies $t_{k}=0$ for $k \neq 2,6$.

For any $C_{i}, C_{j} \in \mathcal{C}$, let $a^{\prime}:=C_{i} \cdot C_{j}=2 a b-a^{2} e$ and $b^{\prime}:=K_{X} \cdot C_{i}=2 a e+a(2 g-2-e)-2 b$.

For any $j \in\{1, \ldots, d\}$, let $t_{k}^{j}$ denote the number of $k$-fold points of $C_{j}$. Since $t_{k}=0$ for $k \neq 2,6$, Lemma 2.5(1) gives

$$
a^{\prime}(d-1)=5 t_{6}^{j}+t_{2}^{j}
$$


The second kind of curves contained in the ramification divisor of $\sigma$ are $D_{j}:=\sigma^{\star}\left(C_{j}^{\prime}\right)$, where $C_{j}^{\prime}$ is the strict transform of $C_{j}$ under the blow up $\tau$. We now calculate the relative proportionality $\operatorname{prop}\left(D_{j}\right)$.

Note that $K_{Y}=\sigma^{\star}(T)$, where $T$ was defined in Lemma 3.2. We also recall that, by (4.2), we have $T \cdot C_{j}^{\prime}=b^{\prime}+\frac{a^{\prime}}{2}+f_{0}^{j}-\frac{t_{2}^{j}}{2}$. Finally, note that $C_{j}^{\prime 2}=C_{j}^{2}-\sum_{k \geq 3} t_{k}^{j}=a^{\prime}-\sum_{k \geq 3} t_{k}^{j}$.

Then $\operatorname{prop}\left(D_{j}\right)=2 D_{j}^{2}-e\left(D_{j}\right)=3 D_{j}^{2}+K_{Y} \cdot D_{j}=3\left(\left(\frac{2^{d-1}}{2^{2}}\right) C_{j}^{\prime 2}\right)+\left(\frac{2^{d-1}}{2}\right)\left(T \cdot C_{j}^{\prime}\right)=$ $2^{d-3}\left(3 a^{\prime}-3 \sum_{k \geq 3} t_{k}^{j}\right)+2^{d-3}\left(2 b^{\prime}+a^{\prime}+2 f_{0}^{j}-t_{2}^{j}\right)=2^{d-3}\left(4 a^{\prime}+2 b^{\prime}-t_{6}^{j}+t_{2}^{j}\right)$.

For the final equality above, we use the fact that $t_{k}=0$ for $k \neq 2,6$. If $Y$ is a ball quotient, then $\operatorname{prop}\left(D_{j}\right)=0$. This gives

$$
4 a^{\prime}+2 b^{\prime}=t_{6}^{j}-t_{2}^{j}
$$

Solving the linear equations (5.1) and (5.2) for $t_{2}^{j}$ and $t_{6}^{j}$, and using the easy combinatorial identity $\sum_{j=1}^{d} t_{k}^{j}=k t_{k}$, we get

$$
t_{2}=\frac{a^{\prime} d^{2}-21 a^{\prime} d-10 b^{\prime} d}{12}, t_{6}=\frac{a^{\prime} d^{2}+3 a^{\prime} d+2 b^{\prime} d}{36} .
$$

If there exists an arrangement $\mathcal{C}$ on $X$ satisfying Assumption 4.3 and having only double and sixfold points such that the associated surface $Y$ is a ball quotient, then $H_{\mathcal{C}}(2)=0$. This gives

$$
16-16 g+d[(2 b-a e)(5 a-2)+4 a(g-1)]+t_{2}=3 t_{6} .
$$

Plugging the values of $t_{2}$ and $t_{6}$ obtained above in (5.4) and simplifying, we get

$$
16-16 g=-d((3 a-1)(2 b-a e)+2 a(g-1)) .
$$

We can rewrite (5.5) as

$$
-16=d[(3 a-1)(2 b-a e)-2 a]+(2 a d-16) g .
$$

Thus by our assumptions, we have

$$
\begin{aligned}
d[(3 a-1)(2 b-a e)-2 a] & \geq d[(3 a-1) a e-2 a]=a d[e(3 a-1)-2] \\
& >0 .
\end{aligned}
$$

Note that $d \geq 4$ by Assumption 2.4. So if $a \geq 2$ or if $a=1, d \geq 8$, then (2ad-16) $g \geq 0$ and thus the right-hand side of (5.6) is a positive number, a contradiction.

Let $a=1$ and $4 \leq d \leq 7$. Then it is easy to directly check that (5.4) is not possible. First note that the largest value of $t_{6}$ is attained when $t_{k}=0$ for $k \neq 6$ and in this case we have $t_{6}=\frac{a^{\prime} d(d-1)}{30}$, by Lemma $2.5(2)$. 
If $Y$ is a ball quotient, then (5.4) holds and we have

$$
\begin{aligned}
0 & =16-16 g+d[(2 b-a e)(5 a-2)+4 a(g-1)]+t_{2}-3 t_{6} \\
& \geq 16-16 g+d(6 b-3 e+4 g-4)-\frac{a^{\prime} d(d-1)}{10} \\
& \geq 16-16 g+4 g d-4 d+(2 b-e)\left(3 d-\frac{d(d-1)}{10}\right) \\
& \geq 16-4 d+4\left(3 d-\frac{d(d-1)}{10}\right), \quad \text { since } d \geq 4, b \geq e \geq 4 .
\end{aligned}
$$

Now it is easy to check that the last term above is positive for $4 \leq d \leq 7$, giving a contradiction.

The above arguments prove the following theorem.

Theorem 5.2. Let $X$ be a ruled surface with $e \geq 4$. There does not exist any transversal arrangement $\mathcal{C}$ on $X$ satisfying Assumption 4.3 such that the associated surface $Y$ is a ball quotient.

Acknowledgement: We thank Piotr Pokora for reading this paper and giving many useful suggestions. We also thank the referees for making several helpful suggestions which substantially improved the paper.

\section{REFERENCES}

[1] W. Barth, C. Peters and A. Van de Ven, Compact complex surfaces, Ergebnisse der Mathematik und ihrer Grenzgebiete (3), 4, Springer-Verlag, Berlin, 1984.

[2] G. Barthel, F. Hirzebruch and T. Höfer, Geradenkonfigurationen und Algebraische Flächen, Aspects of Mathematics, D4, Friedr. Vieweg \& Sohn, Braunschweig, 1987.

[3] T. Bauer, S. Di Rocco, B. Harbourne, J. Huizenga, A. Lundman, P. Pokora and T. Szemberg, Bounded negativity and arrangements of lines, Int. Math. Res. Not. IMRN 2015, no. 19, 9456-9471.

[4] T. Bauer, B. Harbourne, A. Knutsen, A. Küronya, S. Müller-Stach, X. Roulleau and T. Szemberg, Negative curves on algebraic surfaces, Duke Math. J. 162 (2013), no. 10, 1877-1894.

[5] M. Dumnicki, D. Harrer and J. Szpond, On absolute linear Harbourne constants, Finite Fields Appl. 51 (2018), 371-387.

[6] S. Eterović, Logarithmic Chern slopes of arrangements of rational sections in Hirzebruch surfaces, Master Thesis, Pontificia Universidad Católica de Chile, Santiago 2015.

[7] R. Hartshorne, Algebraic geometry, Springer-Verlag, New York, 1977.

[8] J. C. Hemperly, The parabolic contribution to the number of linearly independent automorphic forms on a certain bounded domain, Amer. J. Math. 94 (1972), 1078-1100.

[9] E. Hironaka, Abelian coverings of the complex projective plane branched along configurations of real lines, Mem. Amer. Math. Soc. 105 (1993), no. 502, vi+85 pp.

[10] F. Hirzebruch, Arrangements of lines and algebraic surfaces, in Arithmetic and geometry, Vol. II, Progr. Math., 36, Birkhäuser, Boston, Mass, 113-140 (1983).

[11] F. Hirzebruch, Algebraic surfaces with extremal Chern numbers (based on a dissertation by T. Höfer, Bonn, 1984), Uspekhi Mat. Nauk 40 (1985), no. 4(244), 121-129.

[12] F. Hirzebruch, Singularities of algebraic surfaces and characteristic numbers, in The Lefschetz centennial conference, Part I (Mexico City, 1984), 141-155, Contemp. Math., 58, Amer. Math. Soc., Providence, RI.

[13] F. Klein, Ueber die Transformation siebenter Ordnung der elliptischen Functionen, Math. Ann. 14 (1878), no. 3, 428-471. 
[14] R. Laface and P. Pokora, On the local negativity of surfaces with numerically trivial canonical class, Atti Accad. Naz. Lincei Rend. Lincei Mat. Appl. 29 (2018), no. 2, 237-253.

[15] R. Laface and P. Pokora, Local negativity of surfaces with non-negative Koidara dimension and transversal configurations of curves, To appear in Glasg. Math. J., arXiv:1602.05418,

[16] M. Namba, Branched coverings and algebraic functions, Pitman Research Notes in Mathematics Series, 161, Longman Scientific \& Technical, Harlow, 1987.

[17] R. Pardini, Abelian covers of algebraic varieties, J. Reine Angew. Math. 417 (1991), 191-213. MR1103912

[18] P. Pokora, Harbourne constants and arrangements of lines on smooth hypersurfaces in $\mathbb{P}_{\mathbb{C}}^{3}$, Taiwanese J. Math. 20 (2016), no. 1, 25-31.

[19] P. Pokora, Hirzebruch-type inequalities and plane curve configurations, Internat. J. Math. 28 (2017), no. 2, 1750013, 11 pp.

[20] P. Pokora, Hirzebruch-Kummer covers of algebraic surfaces, Turkish J. Math. 43, 412-421 (2019).

[21] P. Pokora, X. Roulleau and T. Szemberg, Bounded negativity, Harbourne constants and transversal arrangements of curves, Ann. Inst. Fourier (Grenoble) 67 (2017), no. 6, 2719-2735.

[22] P. Pokora and H. Tutaj-Gasińska, Harbourne constants and conic configurations on the projective plane, Math. Nachr. 289 (2016), no. 7, 888-894.

[23] X. Roulleau, Bounded negativity, Miyaoka-Sakai inequality, and elliptic curve configurations, Int. Math. Res. Not. IMRN 2017, no. 8, 2480-2496.

[24] P. Tretkoff, Complex ball quotients and line arrangements in the projective plane, Mathematical Notes, 51, Princeton University Press, Princeton, NJ, 2016.

[25] A. Wiman, Zur Theorie der endlichen Gruppen von birationalen Transformationen in der Ebene, Math. Ann. 48 (1896), no. 1-2, 195-240.

Chennai Mathematical Institute, H1 Sipcot it Park, Siruseri, Kelambakkam 603103, INDIA

E-mail address: krishna@cmi.ac.in

Chennai Mathematical Institute, H1 SipCOT it Park, Siruseri, Kelambakkam 603103, INDIA

E-mail address: adityas@cmi.ac.in 